\title{
Murai反应中芳香酮邻位C-H键活化的区域选择 性的理论研究
}

\author{
唐艳辉 $1^{*}$, 李慧 2,3 , 李龙飞 ${ }^{3}$ \\ 1. 北京服装学院材料科学与工程学院, 北京 100029 \\ 2. 北方民族大学化学与化学工程学院, 银川 750021 \\ 3. 北京化工大学化工资源有效利用国家重点实验室, 北京 100029 \\ *通讯作者, E-mail: clyyanhuitang@bift.edu.cn
}

收稿日期: 2017-02-11; 接受日期: 2017-02-28; 网络版发表日期: 2017-03-24

国家自然科学基金(编号: 21672018)和北京服装学院重点项目(编号: 2014A-01)资助

\begin{abstract}
摘要 钓催化剂 $\mathrm{RuH}_{2}(\mathrm{CO})\left(\mathrm{PPh}_{3}\right)_{3}$ 使 Murai 反应中芳香酮 $\beta$ 位 $\mathrm{C}-\mathrm{H}$ 键的催化活化反应具有极高的产率与选择 性. 本文采用密度泛函(DFT)方法研究了钓配合物催化芳香酮邻位 $\mathrm{C}-\mathrm{H}$ 键活化的反应机理, 剖析了芳香酮 $\mathrm{C}-\mathrm{H}$ 键活化反应中产生区域选择性的原因. 计算结果表明, C-H键的活化位垒为 $1.1 \mathrm{kcal} / \mathrm{mol}$, 从反应动态学角度 很好地解释了该反应的区域选择性. 通过路径 $\mathrm{a}$ 与路径 $\mathrm{b}$ 的比较, 发现 $\mathrm{C}=\mathrm{C}$ 双键更容易插入到 $\mathrm{Ru}-\mathrm{H}$ 键而不是 $\mathrm{Ru}-\mathrm{C}$ 键中. 另外, 无论 $\mathrm{C}-\mathrm{C}$ 键形成 ( $\mathrm{C}-\mathrm{C}$ 活化过程)出现在路径 $\mathrm{a}$ 的烯烃插入基元反应, 还是出现在路径 $\mathrm{b}$ 的还 原消除基元反应, $\mathrm{C}-\mathrm{C}$ 键形成步骤都是整个催化反应的决速步骤. 与路径 $\mathrm{a}$ 和 $\mathrm{b}$ 比较, 反应路径 $\mathrm{c}$ 中 $\mathrm{C}-\mathrm{C}$ 键形成 过程的空间位阻较大, 能垒也更高。
\end{abstract}

关键词 C-H活化, Murai 反应, 区域选择性, 密度泛函理论, 钓催化剂

\section{1 引言}

$\mathrm{C}-\mathrm{H}$ 键的活化与官能化反应是当前催化化学的 研究热点之一 ${ }^{[1]}$. C-H键属于惰性的化学键, 催化活化 $\mathrm{C}-\mathrm{H}$ 键并官能化将 $\mathrm{C}-\mathrm{H}$ 键转化为 $\mathrm{C}-\mathrm{X}$ 键为现代有机合 成提供了一个强有力的合成方法, 实现了化学绿色高 效、原子经济性和环境友好等现代有机合成化学的 理念, 具有重要的实验、理论与应用意义 ${ }^{[2,3]}$. 过去几 十年中, $\mathrm{C}-\mathrm{H}$ 键活化取得了很大进步 ${ }^{[1,4]}$. C-H键的活化 策略包括基于自由基过程的活化、基于卡宾和氮宾 的插入反应、基于过渡金属参与的环金属化活化反
应等 ${ }^{[3]}$. 过渡金属配合物催化活化 $\mathrm{C}-\mathrm{H}$ 键一直是 $\mathrm{C}-\mathrm{H}$ 键活化官能化反应的重要方法 ${ }^{[2,5 \sim 18]}$. 直接区域选择性 地将不活泼的 $\mathrm{C}-\mathrm{H}$ 键转化为 $\mathrm{C}-\mathrm{C}$ 键的反应逐渐取代采 用格氏试剂或有机锂试剂( $\mathrm{RM}, \mathrm{M}=\mathrm{Li} 、 \mathrm{MgX}$ 等)催化 的传统偶联反应, 且越来越受到重视 ${ }^{[7,9 \sim 11,19-21]}$. 除了钯 和铑催化剂外 ${ }^{[6,9]}$, 钉、铱等过渡金属催化剂也能有效 地活化 $\mathrm{C}-\mathrm{H}$ 键形成 $\mathrm{C}-\mathrm{C}$ 键 ${ }^{[10,20-22]}$.

1993年, Murai等 ${ }^{[1,22224]}$ 将烯烃插入到芳香酮上的 邻位 $\mathrm{C}-\mathrm{H}$ 键, 使芳香酮 $\mathrm{C}-\mathrm{H}$ 键活化官能化取得了重大 进展(图 1, 后文中称为Murai 反应). Murai 反应发现了 一类高效的钓催化剂 $\mathrm{RuH}_{2}(\mathrm{CO})\left(\mathrm{PPh}_{3}\right)_{3}$, 在芳香酮 $\beta$ 位

引用格式: 唐艳辉, 李慧, 李龙飞. Murai反应中芳香酮邻位C-H键活化的区域选择性的理论研究. 中国科学: 化学, 2017, 47: 787-793

Tang Y, Li H, Li L. A theoretical research on the regioselectivity for ortho C-H activation of aromatic ketone in Murai reaction. Sci Sin Chim, 2017, 47: 787-793, doi: 10.1360/N032017-00023 


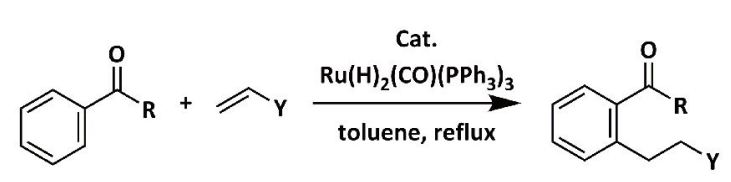

图 1 芳香酮邻位 $\mathrm{C}-\mathrm{H}$ 键与烯烃加成的Murai反应 $(\mathrm{R} 、 \mathrm{Y}$ 代 表取代基)

C-H键的催化活化中, 具有产率高、选择性强、操作 简单等优点. 他们也发现不同的芳香酮底物结构, 邻位 $\mathrm{C}-\mathrm{H}$ 键的断裂具有不同的活性与选择性, 且加成产物 的结构与反应物芳香酮结构有着密切的关系 ${ }^{[25 ~ 27]}$. 在 Murai反应之后, 大量过渡金属化合物高选择性地催 化活化官能化 $\mathrm{C}-\mathrm{H}$ 键的理论与实验研究被报道 ${ }^{[1,22,28]}$. 以前对Murai反应的区域选择性的理论研究表明, $\beta$ 位 碳原子与羰基形成平面“ $U$ 弯区”最低未占分子轨道 (LUMO)共轭结构是进行芳香酮邻位 $\mathrm{C}-\mathrm{H}$ 键活化反应 的必要条件. 在满足上述条件的情况下, 邻位C-H活化 发生在芳香环上正电性较强的邻位 $\mathrm{C}$ 原子上 ${ }^{[29]}$. 值得 注意的是, 也有实验报道在一些催化体系中, 如(PCP)Ir 蟹形配合物催化体系中, 芳香酮的“U弯区”螯合效应 并不是邻位 $\mathrm{C}-\mathrm{H}$ 活化的必要条件 ${ }^{[30]}$.

如图2所示, Murai等提出此C-H键活化加成反应 的可能机理. 首先经过钓催化剂与芳香酮 ${ }^{[1,4]}$ 共轭结构 $\mathrm{C}=\mathrm{O}$ 的成键, 1,2-氢迁移造成 $\mathrm{C}-\mathrm{H}$ 键的断裂, 然后烯烃 插入 $\mathrm{C}-\mathrm{C}$ 键生成最终产物 ${ }^{[22]}$. 探索 $\mathrm{C}-\mathrm{H}$ 键选择性活化 转化的化学规律, 是当前催化化学、有机化学和物理 化学研究的重要科学问题. 虽然实验与理论化学家对 该反应的反应机理进行了一些研究, 但该反应的机理 中, 反应的区域选择性的本质并不清晰. 本文采用密度 泛函(DFT) 方法研究了钓配合物 $\mathrm{Ru}(\mathrm{CO})\left(\mathrm{PPh}_{3}\right)$ 催化芳 香酮邻位 $\mathrm{C}-\mathrm{H}$ 键活化的反应机理, 剖析了芳香酮 $\mathrm{C}-\mathrm{H}$ 键反应的区域选择性的本质. 此外, 我们对 $\mathrm{Ru}\left(\mathrm{PPh}_{3}\right)_{2}$ 催化芳香酮 $\beta$ 位 $\mathrm{C}-\mathrm{H}$ 键活化的机制也进行了相关的 研究.

\section{2 计算方法}

本研究对钓催化剂及反应通道中各驻点结构都采 用了全模型, 苯乙酮为酮反应物, 丙烯为烯烃底物. 理 论计算采用 Gaussian09程序 ${ }^{[31]}$, DFT下的 $\omega$ B $97 \mathrm{xd} / \mathrm{BSI}$ 进行计算 ${ }^{[32]}$. 其中混合基组(BSI)对过渡金属中心采用 基于有效核势能近似(ECP)的LANL2DZ基组, 其他原

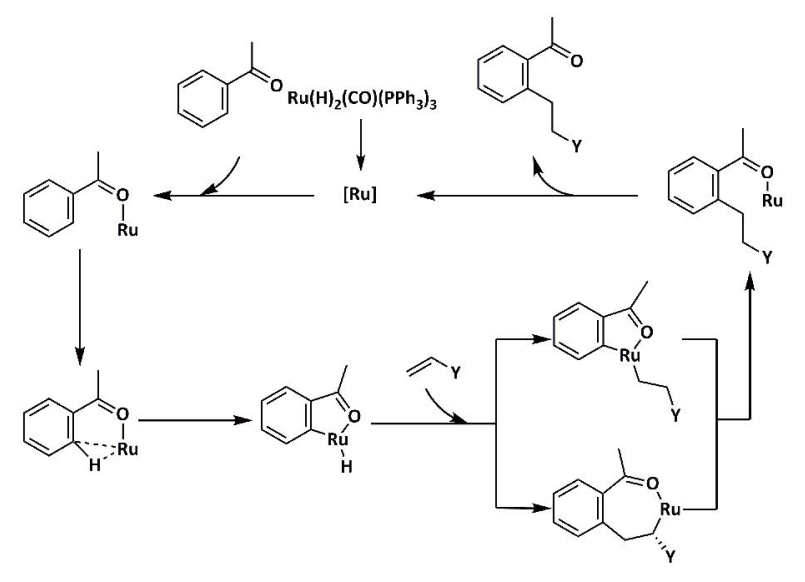

图 $2 \mathrm{RuH}_{2}(\mathrm{CO})\left(\mathrm{PPh}_{3}\right)_{3}$ 催化芳香酮邻位 $\mathrm{C}-\mathrm{H}$ 键活化反应机 理(Y代表取代基)

子采用 $6-31++\mathrm{G}^{*}$ 基组. 在 $\omega B$ B $97 \mathrm{xd} / \mathrm{BSI}$ 水平下优化得 到了苯乙酮 $\mathrm{C}-\mathrm{H}$ 键活化和烯烃插入反应催化循环关 键步骤的各中间体、过渡态和产物的几何构型, 对 所有驻点进行了振动分析, 以确认其中过渡态有且只 有一个虚频. 此外, 所有的能量均采用溶剂化模型 D (SMD), 在甲苯溶液中进行了能量校正. 如没有特别说 明, 能量描述均采用自由能 $G$ (气相条件下, $T=298.15$ $\mathrm{K}, 1 \mathrm{~atm})$, 后文中自由能图中能量数值分别代表自由 能、零点能校对后势能和溶剂化效应势能.

\section{3 结果与讨论}

如图3所示, 催化剂前体 $\mathrm{RuH}_{2}(\mathrm{CO})\left(\mathrm{PPh}_{3}\right)_{3}$ 首先生 成零价二配位的催化活性中间体 $\mathrm{Ru}(\mathrm{CO})\left(\mathrm{PPh}_{3}\right)(\mathbf{1})$. 苯 乙酮 (acetophenone)通过羰基氧和邻位苯环上的 $\mathrm{C}-\mathrm{H}$ 键与金属中心 $\mathrm{Ru}$ 原子配位, 形成双齿的钓苯乙酮配 合物 $\mathrm{Ru}(\mathrm{CO})\left(\mathrm{PPh}_{3}\right)\left(\mathrm{PhCOCH}_{3}\right)(2)$. 与此同时, 苯乙酮配 体上的 $\mathrm{C}^{1}-\mathrm{H}^{1}$ 键被活化, 发生氧化加成反应, 经过渡态 TS2-3发生C-H断裂, 生成 Ru(II)中间体3. 本研究以丙 烯(Sub2)作为烯烃底物, Sub2 与 3 因配位方式不同会 生成两种配位中间体 $4 \mathrm{a}$ 和 $4 \mathrm{c}$. 对 $4 \mathrm{a}$ 而言, 存在两种不 同的 $\mathrm{C}=\mathrm{C}$ 双键插入方式, 从而产生两种不同的反应路 径: 路径 $\mathrm{a}$ 是 $\mathrm{C}^{2}=\mathrm{C}^{3}$ 双键插入到 $\mathrm{Ru}-\mathrm{H}^{1}$ 键活化 $\rightarrow$ 还原消 除反应 ( $C^{1}-C^{2}$ 键形成过程); 路径 $b$ 是 $C^{2}=C^{3}$ 双键插入到 $\mathrm{Ru}-\mathrm{C}^{1}$ 键 $\left(\mathrm{C}^{1}-\mathrm{C}^{2}\right.$ 键形成过程 $) \rightarrow$ 烷基负离子与氢负离子 的还原消除反应(氢转移过程). 对 $4 \mathrm{c}$ 而言, $\mathrm{C}=\mathrm{C}$ 双键以 2,1-插入的方式进行. 


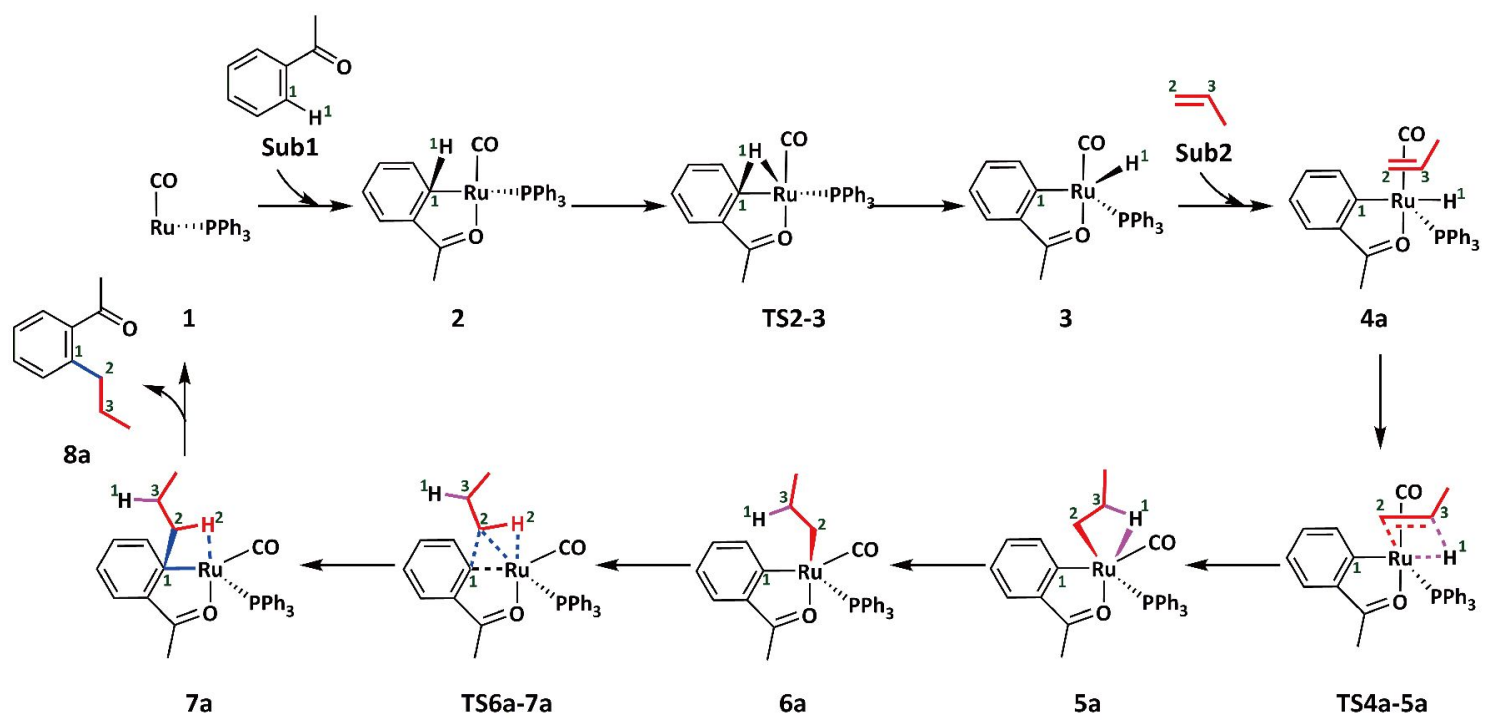

图 $3 \mathrm{RuH}_{2}(\mathrm{CO})\left(\mathrm{PPh}_{3}\right)_{3}$ 催化芳香酮邻位 $\mathrm{C}-\mathrm{H}$ 键活化反应机理(路径a)

\subsection{C-H键活化过程}

从催化剂前体 $\mathrm{RuH}_{2}(\mathrm{CO})\left(\mathrm{PPh}_{3}\right)_{3}$ 可以得到二配位 的反应活性中间体 $\mathrm{Ru}(\mathrm{CO})\left(\mathrm{PPh}_{3}\right)(\mathbf{1})$, 其中 $\mathrm{Ru}$ 为 0 价. 如图4所示, 底物苯乙酮中的羰基氧原子与活性中间 体1中的钓中心原子配位, 同时邻位 $\left(\mathrm{C}^{1}\right) \mathrm{C}-\mathrm{H}$ 键中 $\mathrm{H}^{1}$ 原 子与钉原子形成抓氢作用. 此配位过程形成四配位的 中间体 2, 并且释放 $19.1 \mathrm{kcal} / \mathrm{mol}$ 能量, 表明反应非常容 易进行. 值得注意的是, 形成抓氢键后 $\mathrm{C}-\mathrm{H}$ 键被活化, 其键长从游离态的 $1.085 \AA$ 增加到结合态(中间体2)中 的 $1.175 \AA ; \mathrm{C}$ 与 $\mathrm{H}$ 的原子极性张量(APT)电荷从 $-0.02 \mathrm{e}$, $0.03 \mathrm{e}$ 分别变化到 $0.70 \mathrm{e},-0.18 \mathrm{e}$. 经过渡态 TS2-3发生氧 化加成反应, $\mathrm{C}-\mathrm{H}$ 键断裂形成五配位的中间体 3, C-H距 离增加为 $2.476 \AA . \mathrm{Ru}$ 从 0 价变化到 +2 价, 此时 $\mathrm{C}^{1} \mathrm{APT}$ 电 荷为 $0.32 \mathrm{e}, \mathrm{H}^{1} \mathrm{APT}$ 电荷为 $-0.16 \mathrm{e}$. 从图 4 中可以看出, 过 渡态TS2-3 的能量相较于中间体2仅高出 $1.1 \mathrm{kcal} / \mathrm{mol}$, 而中间体3 的生成过程中释放能量 $29.4 \mathrm{kcal} / \mathrm{mol}$, 这表 明苯乙酮上的邻位 C-H断裂非常容易, 而且五配位的 二价钓化合物更加稳定.

\section{2 氢转移/C-C形成反应路径(路径a)}

如图5所示, 丙烯(Sub2)中的 $\mathrm{C}=\mathrm{C}$ 双键与 $\mathrm{Ru}$ 中心原 子配位, 同时 $\mathrm{H}^{1}$ 异构化到 $\mathrm{C}^{1}$ 原子的反位位置, 最终形成 具有六配位的正八面体中间体 $\mathbf{4 a}$, 烯烃配位过程释放 能量 $9.9 \mathrm{kcal} / \mathrm{mol}$. 然后, $\mathrm{H}^{1}$ 迁移到 $\mathrm{C}^{3}$ 上完成氢迁移过 程, 形成具有抓氢效应的中间体 5a. $C=C$ 双键经由过渡

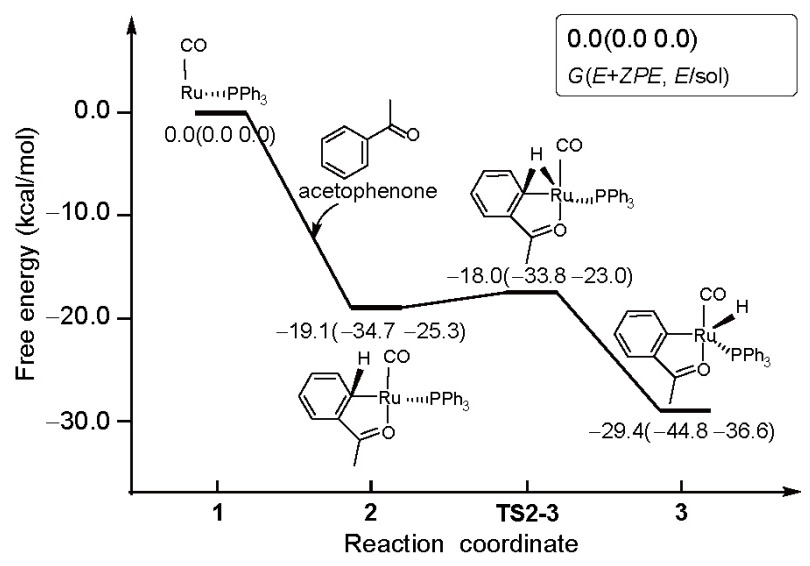

图 $4 \mathrm{Ru}(\mathrm{CO})\left(\mathrm{PPh}_{3}\right)$ 1催化芳香酩邻位 $\mathrm{C}-\mathrm{H}$ 键活化基元反应 步骤自由能图

态TS4a-5a插入到 Ru-H键, 反应能垒为 $4.0 \mathrm{kcal} / \mathrm{mol}, \mathbf{5 a}$ 较4a更稳定. 5a异构化到中间体6a, 形成正丙基配体.

接下来进行的是 $\mathrm{C}-\mathrm{C}$ 成键反应, 即还原消除反应. $\mathrm{C}^{2}$ 向 $\mathrm{C}^{1}$ 移动形成 $\mathrm{C}-\mathrm{C}$ 键, 同时 $\mathrm{Ru}-\mathrm{C}^{2}$ 键断裂生成中间体 $7 \mathbf{a}$, 此过程的过渡态为 TS6a-7a, 能垒为 $23.1 \mathrm{kcal} / \mathrm{mol}$. 最后, 产物8被释放, 同时重新产生催化剂活性中间体 OB, 完成催化循环. 总反应释放能量 $14.0 \mathrm{kcal} / \mathrm{mol}$, 能 量跨度 $(6 \mathbf{a}$ 至TS6a-7a) 为 $23.1 \mathrm{kcal} / \mathrm{mol}$.

\section{$3.3 \mathrm{C}-\mathrm{C}$ 形成/氢转移路径(路径b)}

与反应路径 $\mathrm{a}$ 不同的是, 路径 $\mathrm{b}$ 首先是配位烯烃对 
$\mathrm{Ru}-\mathrm{C}^{1}$ 键的插入 $\left(\mathrm{C}^{1}-\mathrm{C}^{2}\right.$ 键生成反应 $)$, 然后是 $\mathrm{H}^{1}$ 原子迁 移(还原消除反应). 如图6所示, Sub2 中的 $C^{2}$ 与 Sub1 中 的 $\mathrm{C}^{1}$ 成键, 形成四元环的中间体 $\mathbf{5 b}$. 此过程的过渡态 为 TS4a-5b, 能垒为 $27.8 \mathrm{kcal} / \mathrm{mol}$, 远高于 $\mathrm{a}$ 路径中 $\mathrm{C}^{2}=\mathrm{C}^{3}$ 双键插入到 $\mathrm{Ru}-\mathrm{H}^{1}$ 键的能垒 $4.0 \mathrm{kcal} / \mathrm{mol}$.
在还原消除反应中, $\mathrm{H}^{1}$ 转移到 $\mathrm{C}^{3}$ 上, $\mathrm{Ru}$ 原子从 +2 价被还原为 0 价, 生成与产物具有配位结合形态的三 配位产物 6b. 此过程的过渡态 TS5b-6b, 能垒为 20.6 $\mathrm{kcal} / \mathrm{mol}$, 略低于路径 $\mathrm{a}$ 中还原释放的反应位垒 $(23.1$ $\mathrm{kcal} / \mathrm{mol})$. 最后, 产物 8 脱除并重新生成催化剂活性中

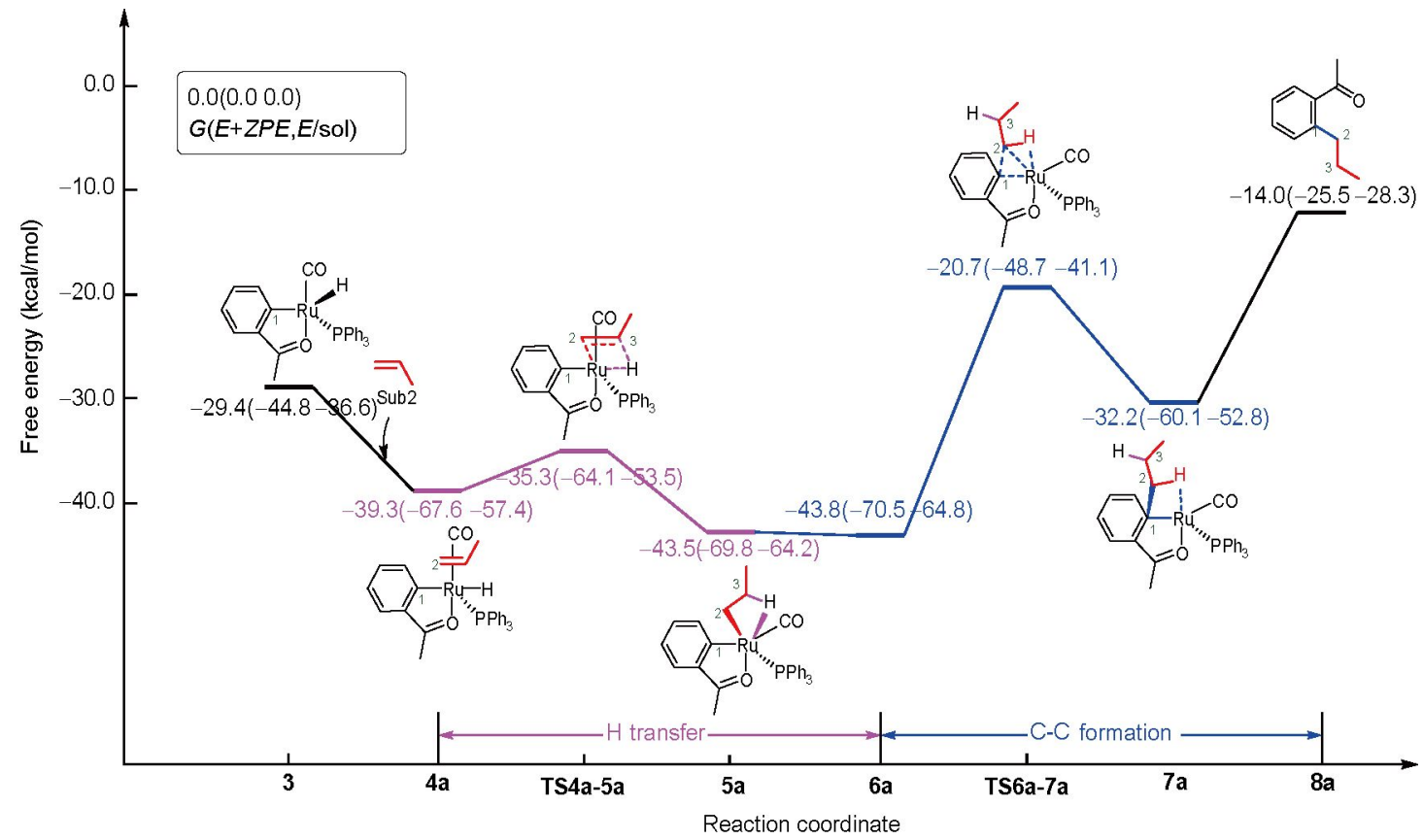

图 5 路径 $\mathrm{a}$ 中氢转移/C-C键形成基元反应步骤自由能图

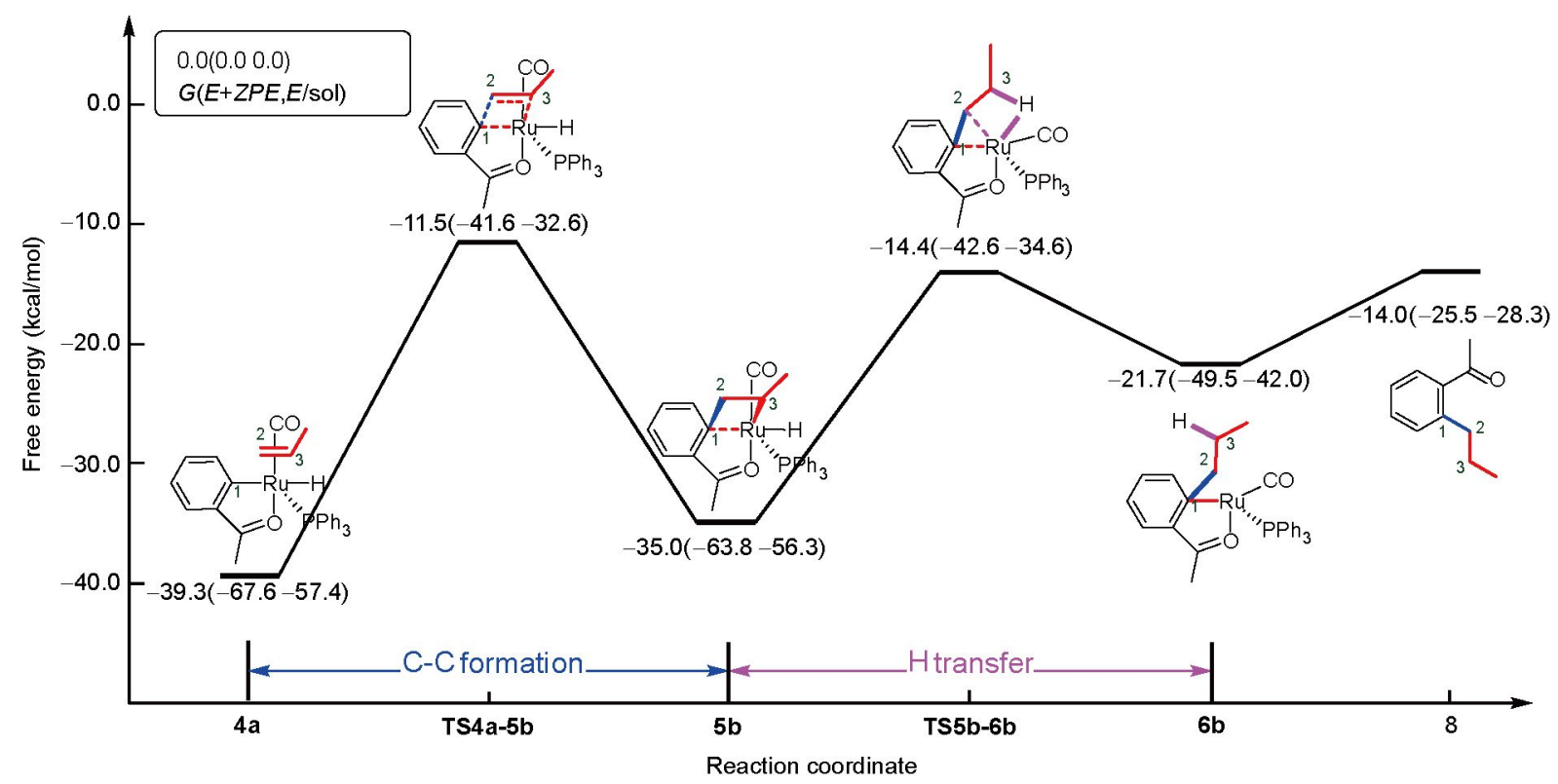

图 6 路径 $\mathrm{b}$ 中 $\mathrm{C}-\mathrm{C}$ 键形成/氢转移基元反应步骤自由能图 


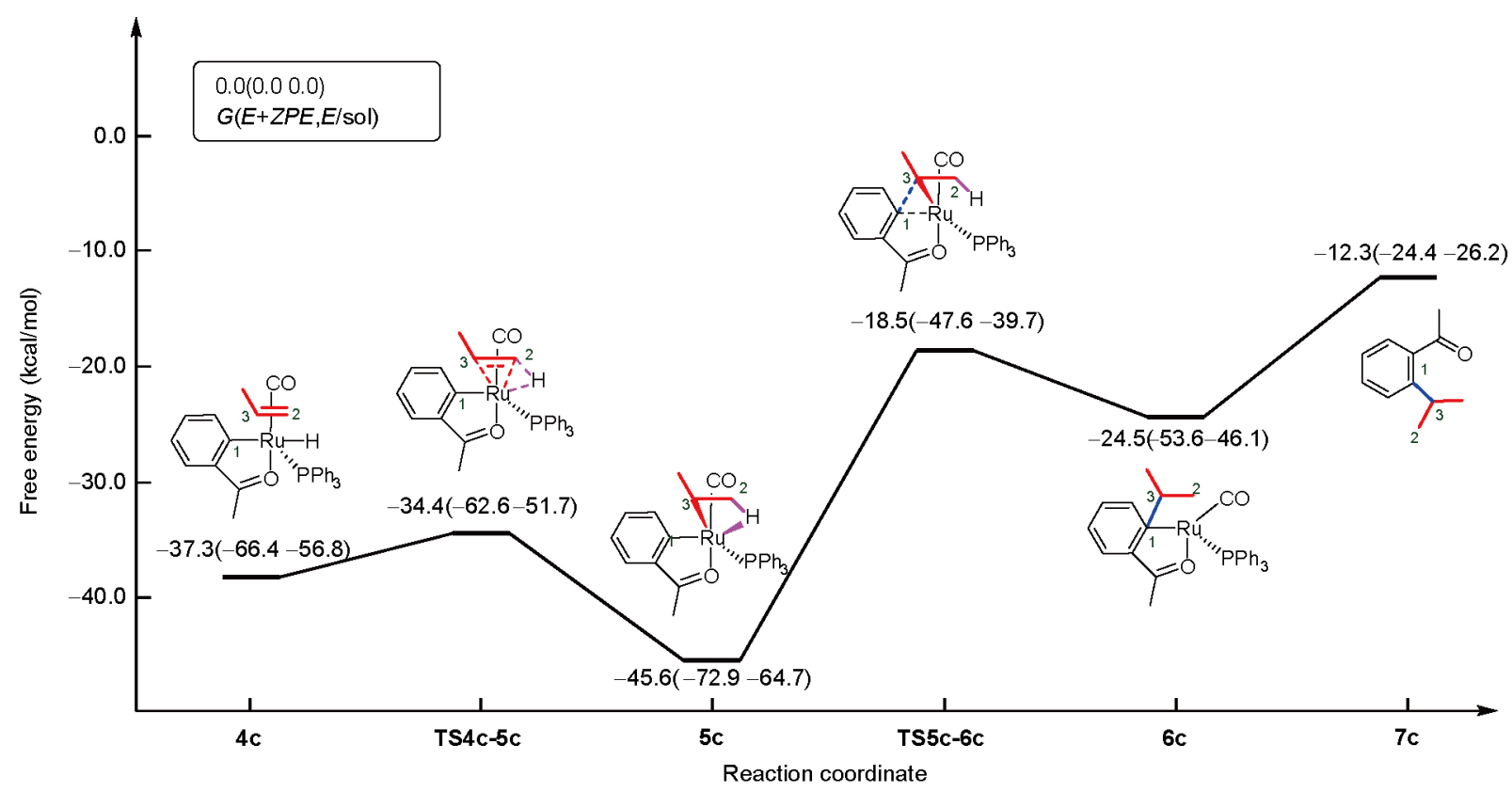

图 7 路径 $\mathrm{c}$ 中氢转移/C-C键形成基元反应步骤自由能图

间体 $\mathbf{1}$, 完成催化循环反应. 由于路径 $\mathrm{a}, \mathrm{b}$ 的产物相同, 总反应释放能量也相同 $(14.0 \mathrm{kcal} / \mathrm{mol})$. 从能量跨度来 看, 路径 $\mathrm{b}$ 为 $27.8 \mathrm{kcal} / \mathrm{mol}$ (4b 至 TA4a-5b), 而路径 $\mathrm{a}$ 为 $23.1 \mathrm{kcal} / \mathrm{mol}$, 路径 $\mathrm{a}$ 为优势反应路径.

\section{4 氢转移/2,1-C-C插入反应路径(路径c)}

由于丙烯的配位方式最终决定了产物的构型, 考 虑路径 $a, b$ 中丙烯的配位方式外, 也考虑了另一种丙烯 配位方式. 如图7所示, 丙烯与催化剂金属中心 Ru配位 后形成正八面体中间体 $4 \mathbf{c}$, 其能量较 $4 \mathbf{a}$ 高 $2.0 \mathrm{kcal} / \mathrm{mol}$. 丙烯中的 $\mathrm{C}^{2}=\mathrm{C}^{3}$ 双键插入 $\mathrm{Ru}-\mathrm{H}^{1}$ 键过程中, 经过渡态 TS4c-5c 形成具有抓氢效应的中间体结构 $5 \mathrm{c}$, 此过程 释放能量 $8.3 \mathrm{kcal} / \mathrm{mol}$, 反应能垒为 $2.9 \mathrm{kcal} / \mathrm{mol}$, 表明 $\mathrm{C}^{2}=\mathrm{C}^{3}$ 双键可以很容易以 2,1 插入的方式插入到 $\mathrm{Ru}-\mathrm{H}^{1}$ 键.

在之后的还原消除反应中, $C^{2}$ 向Sub1 中的 $C^{1}$ 移动 形成 $\mathrm{C}-\mathrm{C}^{2}$ 键, 同时 $\mathrm{Ru}-\mathrm{C}^{1}$ 键断裂生成 $6 \mathrm{c}$ 中间体. 此过 程的过渡态为 $\mathbf{T S 5 c - 6 c}$, 能垒为 $27.1 \mathrm{kcal} / \mathrm{mol}$. 最后, 产 物 7c被释放, 同时产生催化剂活性中间体 1 , 完成催化
循环。总反应释放能量 $12.3 \mathrm{kcal} / \mathrm{mol}$, 能量跨度 $\mathbf{5} \mathbf{5 c}$ 至 TS5c-6c) 为 $27.1 \mathrm{kcal} / \mathrm{mol}$, 比路径 $\mathrm{a}$ 高.

此外, $\mathrm{Ru}\left(\mathrm{PPh}_{3}\right)_{2}$ 和 $\mathrm{Ru}(\mathrm{CO})\left(\mathrm{PPh}_{3}\right)$ 催化芳香酮 $\beta$ 位 $\mathrm{C}-\mathrm{H}$ 键活化的反应的机理类似, $\mathrm{C}-\mathrm{H}$ 键活化/氢转移/烯 烃1,2-插入为优势反应路径(结合机制的反应机理和 自由能面图见网络版补充材料).

\section{4 结论}

本研究采用DFT方法研究了 $\mathrm{RuH}_{2}(\mathrm{CO})\left(\mathrm{PPh}_{3}\right)_{3}$ 催化 芳香酮 $\beta$ 位 $\mathrm{C}-\mathrm{H}$ 键活化官能化反应的催化机理. 计算结 果表明, C-H键活化/氢转移/烯烃1,2-插入为优势反应 路径, 其中 $\mathrm{C}-\mathrm{H}$ 键的活化位垒为 $1.1 \mathrm{kcal} / \mathrm{mol}$, 这很好地 解释了该反应的区域选择性; 速控步反应位垒为 23.1 $\mathrm{kcal} / \mathrm{mol}$, 说明 $\mathrm{C}=\mathrm{C}$ 双键更容易插入到 $\mathrm{Ru}-\mathrm{H}$ 键而不是 $\mathrm{Ru}-\mathrm{C}$ 键中. 此外, 在整个反应循环中, 不管 $\mathrm{C}-\mathrm{C}$ 键形成 过程出现在路径 $\mathrm{a}$ 的烯烃插入还是路径 $\mathrm{b}$ 的还原消除反 应中, 都是整个反应循环的决速步骤. 烯烃1,2-插入相 较于 2,1 -插入更容易, 这是因为 $\mathbf{5 a}$ 中的 $\mathrm{C}^{3}$ 连接一个甲 基, 而 $5 \mathrm{c}$ 中的 $\mathrm{C}^{3}$ 连接两个甲基而具有更大的空间位阻.

\section{补充材料}

本文的补充材料见网络版 chemen.scichina.com. 补充材料为作者提供的原始数据, 作者对其学术质量和内容负责. 


\section{参考文献}

李洋, 施章杰.中国科学: 化学, 2016, 46: 579-587

2 刘杰, 朱庆仁, 陶杨, 张袖丽. 科学通报, 2015, 60: 2918-2926

3 Chen G, Shi ZJ. Natl Sci Rev, 2014, 1: 172-175

4 许泽君, 焦宁. 中国科学: 化学, 2013, 43: 1121-1134

5 占贝贝, 刘斌, 胡芳, 史炳锋. 科学通报, 2015, 60: 2907-2917

6 Topczewski JJ, Sanford MS. Chem Sci, 2015, 6: 70-76

7 Yan G, Wu X, Yang M. Org Biomol Chem, 2013, 11: 5558-5578

8 牛坡, 温庭斌. 中国科学: 化学, 2011, 41: 943-955

9 Colby DA, Bergman RG, Ellman JA. Chem Rev, 2010, 110: 624-655

10 Arockiam PB, Bruneau C, Dixneuf PH. Chem Rev, 2012, 112: 5879-5918

11 Kakiuchi F, Murai S. Acc Chem Res, 2002, 35: 826-834

12 Li B, Dixneuf PH. Chem Soc Rev, 2013, 42: 5744-5767

13 Jiang TS, Wang GW. J Org Chem, 2012, 77: 9504-9509

14 Nakamura E, Yoshikai N. J Org Chem, 2010, 75: 6061-6067

15 Esteruelas MA, López AM. Organometallics, 2005, 24: 3584-3613

16 Yamaguchi J, Yamaguchi AD, Itami K. Angew Chem Int Ed, 2012, 51: 8960-9009

17 Rouquet G, Chatani N. Angew Chem Int Ed, 2013, 52: 11726-11743

18 Jiang YY, Man X, Bi S. Sci China Chem, 2016, 59: 1448-1466

19 Song G, Wang F, Li X. Chem Soc Rev, 2012, 41: 3651-3678

20 Yang L, Huang H. Catal Sci Technol, 2012, 2: 1099-1112

21 Kakiuchi F, Kochi T, Mizushima E, Murai S. J Am Chem Soc, 2010, 132: 17741-17750

22 Murai S, Kakiuchi F, Sekine S, Tanaka Y, Kamatani A, Sonoda M, Chatani N. Nature, 1993, 366: 529-531

23 Murai S, Kakiuchi F, Sekine S, Tanaka Y, Kamatani A, Sonoda M, Chatani N. ChemInform, 2010, doi: 10.1002/chin.199442300

24 Kakiuchi F, Sekine S, Tanaka Y, Kamatani A, Sonoda M, Chatani N, Murai S. Bull Chem Soc Jpn, 1995, 68: 62-83

25 Oi S, Fukita S, Hirata N, Watanuki N, Miyano S, Inoue Y. Org Lett, 2001, 3: 2579-2581

26 Ackermann L, Althammer A, Born R. Angew Chem Int Ed, 2006, 45: 2619-2622

27 Özdemir I, Demir S, Cetinkaya B, Gourlaouen C, Maseras F, Bruneau C, Dixneuf PH. J Am Chem Soc, 2008, 130: 1156-1157

28 Matsubara T, Koga N, Musaev DG, Morokuma K. Organometallics, 2000, 19: 2318-2329

29 Lei M, Feng W, Du H, Xu Z. Sci China Chem, 2000, 43: 412-420

30 Zhang X, Kanzelberger M, Emge TJ, Goldman AS. J Am Chem Soc, 2004, 126: 13192-13193

31 Frisch MJ, Trucks GW, Schlegel HB, Scuseria GE, Robb MA, Cheeseman JR, Scalmani G, Barone V, Mennucci B, Petersson GA, Nakatsuji H, Caricato M, Li X, Hratchian HP, Izmaylov AF, Bloino J, Zheng G, Sonnenberg JL, Hada M, Ehara M, Toyota K, Fukuda R, Hasegawa J, Ishida M, Nakajima T, Honda Y, Kitao O, Nakai H, Vreven T, Montgomery JA Jr., Peralta JE, Ogliaro F, Bearpark M, Heyd JJ, Brothers E, Kudin KN, Staroverov VN, Keith T, Kobayashi R, Normand J, Raghavachari K, Rendell A, Burant JC, Iyengar SS, Tomasi J, Cossi M, Rega N, Millam JM, Klene M, Knox JE, Cross JB, Bakken V, Adamo C, Jaramillo J, Gomperts R, Stratmann RE, Yazyev O, Austin AJ, Cammi R, Pomelli C, Ochterski JW, Martin RL, Morokuma K, Zakrzewski VG, Voth GA, Salvador P, Dannenberg JJ, Dapprich S, Daniels AD, Farkas O, Foresman JB, Ortiz JV, Cioslowski J, Fox DJ. GAUSSIAN 09 (Revision B.01). Wallingford CT: Gaussian, Inc., 2010

32 Chai JD, Head-Gordon M. Phys Chem Chem Phys, 2008, 10: 6615-6620 


\title{
A theoretical research on the regioselectivity for ortho $\mathrm{C}-\mathrm{H}$ activation of aromatic ketone in Murai reaction
}

\author{
Yanhui Tang ${ }^{1 *}$, Hui $\mathrm{Li}^{2,3}$, Longfei $\mathrm{Li}^{3}$ \\ ${ }^{1}$ School of Materials Science \& Engineering, Beijing Institute of Fashion Technology, Beijing 100029, China \\ ${ }^{2}$ College of Chemistry and Chemical Engineering, Beifang University of Nationalities, Yinchuan 750021, China \\ ${ }^{3}$ State Key Laboratory of Chemical Resource Engineering, Beijing University of Chemical Technology, Beijing 100029, China \\ *Corresponding author (email: clyyanhuitang@bift.edu.cn)
}

\begin{abstract}
RuH}_{2}(\mathrm{CO})\left(\mathrm{PPh}_{3}\right)$ is an important catalyst for Murai reaction, which brings a very high regioselectivity in ortho $\mathrm{C}-\mathrm{H}$ activation of aromatic ketone. In this work, we studied the mechanism of ruthenium complexes catalyzed aromatic ketone ortho $\mathrm{C}-\mathrm{H}$ activation reaction by density functional method (DFT) method, and explained the regioselectivity by designing three different reaction processes, path $\mathrm{a}$, path $\mathrm{b}$ and path $\mathrm{c}$. Calculated results show that the barrier for $\mathrm{C}-\mathrm{H}$ activation is $1.1 \mathrm{kcal} / \mathrm{mol}$, which explains well the regioselectivity. In the comparison of path a and path $\mathrm{b}$, it is found that the $\mathrm{C}=\mathrm{C}$ double bond is easier to insert to the $\mathrm{Ru}-\mathrm{H}$ bond than the $\mathrm{Ru}-\mathrm{C}$ bond. In addition, the process of $\mathrm{C}-\mathrm{C}$ bond formation ( $\mathrm{C}-\mathrm{C}$ activation) is the rate determining step in the reaction cycle no matter it appears in the olefin insertion step in path a or in the reductive elimination step in path $b$. The comparison of the path $c$ with path a and path $b$ suggests that the steric hindrance in the formation of $\mathrm{C}-\mathrm{C}$ bond in path $\mathrm{c}$ is much bigger than that in path a and path $\mathrm{b}$, which results in a higher energy barrier in this step.
\end{abstract}

Keywords: $\mathrm{C}-\mathrm{H}$ activation, Murai reaction, regioselectivity, DFT, ruthenium complex

doi: $10.1360 / \mathrm{N} 032017-00023$ 